\title{
THE CONNER-FLOYD MAP FOR FORMAL $A$-MODULES
}

\author{
KEITH JOHNSON
}

\begin{abstract}
A generalization of the Conner-Floyd map from complex cobordism to complex $K$-theory is constructed for formal $A$-modules when $A$ is the ring of algebraic integers in a number field or its $p$-adic completion. This map is employed to study the Adams-Novikov spectral sequence for formal $A$-modules and to confirm a conjecture of D. Ravenel.
\end{abstract}

0. Introduction. Let BP be the spectrum representing Brown-Peterson cohomology with respect to a prime $p$ and let $E$ be the Adams summand of complex $K$-theory with respect to this prime. The BP version of the Conner-Floyd map is a map of spectra $\mathrm{BP} \rightarrow E$ which induces a natural equivalence

$$
\mathrm{BP}_{*} X \otimes_{\mathrm{BP} *} E_{*} \simeq E_{*} X .
$$

In particular this induces an isomorphism

$$
E_{*} \otimes_{\mathrm{BP}}, \mathrm{BP}_{*} \mathrm{BP} \otimes_{\mathrm{BP} *} E_{*} \simeq E_{*} E
$$

and so provides a way of computing the Hopf algebra of stable co-operations for $E$ from those for BP. Using this one can obtain a description of $E_{*} E$ similar to that for $K_{*} K$ contained in [AHS]. The study of $\mathrm{BP}$ and the computation of $\mathrm{BP} * \mathrm{BP}$ are based on a study of formal group law, in particular the $p$-typical formal group law.

In [R1] Ravenel studied a generalization of this situation where the formal group law is replaced by a formal $A$-module where $A$ is the ring of integers in an algebraic number field $K$ or its $p$-adic completion. The purpose of the present paper is to describe the corresponding generalization of the map $\left(\mathrm{BP}_{*}, \mathrm{BP}_{*} \mathrm{BP}\right) \rightarrow\left(E_{*}, E_{*} E\right)$ induced by the Conner-Floyd map, and to compute the generalization of $E_{*} E$. This is of interest because it provides some information about a conjecture (3.10) made in [R1]. This conjecture concerned the value of a certain Ext group $\operatorname{Ext}_{V_{A} T}\left(V_{A}, V_{A}\right)$ when $K$ is an extension of the field $Q_{p}$ of $p$-adic numbers. Here $\left(V_{A}, V_{A} T\right)$ is the Hopf algebroid corresponding to the $A$-typical formal $A$ module. This group was conjectured to be, up to small factor, $A / J_{n(q-1)}^{A}$. Here $J_{n(q-1)}^{A}$ is the ideal of $A$ generated by the elements of the form $a^{n}-1$ for units $a$ of $A$ congruent to $1 \bmod (\pi)$ and $(\pi)$ is the unique prime ideal in $A$. We will show, using the generalization of the Conner-Floyd map, that $A / J_{n(q-1)}^{A}$ occurs as $E_{1}^{1,0}$ in the chromatic spectra sequence for formal $A$-modules [R1, Lemma 2.10] and that the small factor in the conjecture is contributed by the nontriviality of the differential $d_{1}$ originating from this group. We will analyze this differential and show that it is nonzero for $A$

Received by the editors June 27, 1986.

1980 Mathematics Subject Classification (1985 Revision). Primary 55T25; Secondary 55N22, 14 L05. 
the ring of integers in a totally ramified extension of degree a power of $p, p^{l}$, of the field obtained by adjoining $p$ th roots of unity to $Q_{p}$, but zero for all other $A$ (thus the small factor is nontrivial for $K=Q_{2}(p=2, l=0)$ and $K=Q_{3}[\sqrt{-3}]$ $(p=3, l=0)$, the two special cases considered in $[\mathbf{R} 1])$. For $A$ of this type we also identify those dimensions in which the small factor is nontrivial and give an estimate of its size in terms of that of $J^{A}$ and the number of roots of unity of $p$ th power order contained in $A$.

The paper is organized as follows:

In $\S 1$ we define the Hopf algebroid $\left(E_{A}, E_{A} T\right)$ which is our generalization of $\left(E_{*}, E_{*} E\right)$ and a map of Hopf algebroids $\left(V_{A}, V_{A} T\right) \rightarrow\left(E_{A}, E_{A} T\right)$. We also define a second map of Hopf algebroids

$$
\left(E_{A}, E_{A} T\right) \rightarrow\left(K\left[v, v^{-1}\right], K\left[u, u^{-1}, v, v^{-1}\right]\right)
$$

and study $E_{A} T$ by studying the image of the composition map

$$
\Phi: V_{A} T \rightarrow K\left[u, u^{-1}, v, v^{-1}\right] .
$$

In $\S 2$, we define a certain subalgebra $C$ of the ring of Laurent polynomials. This algebra consists of those Laurent polynomials satisfying a certain integrality condition and is related to similar rings studied by Georg Pólya and Alexander Ostrowski over 60 years ago. We show that in dimension 0 the image of $\Phi$ is equal to $C$ and then show that the map $\left(E_{A} T\right)_{0} \rightarrow C$ is an isomorphism. Using this description of $E_{A} T$ we compute the Ext group $\operatorname{Ext}_{E_{A} T}\left(E_{A}, E_{A}\right)$ in $\S 3$ and show that this is isomorphic, via a $v_{1}$ local change of rings theorem, to the group $\operatorname{Ext}_{V_{A} T}\left(V_{A}, M^{1}\right)$ in the chromatic spectral sequence. We then do the number theory necessary to identify those extensions for which the relevant differential is nontrivial.

I would like to thank Doug Ravenel for his comments on a preliminary version of this paper. The calculations in $\S 3$ (Corollaries 27 and 28) in particular owe a great deal to his suggestions.

1. The map $\Phi$ and generators for $\operatorname{Im} \Phi$. We will suppose that $A$ is the ring of integers in a finite extension $K$ of $Q_{p}$, the $p$-adic numbers, with maximal ideal $(\pi)$ and residue field $F_{q}$. If $q=p^{f}$, and $e$ is the ramification index of $p$ in $A$, i.e. $\left(\pi^{e}\right)=(p)$, then $e \cdot f$ is the index of the extension [CF].

In analogy with definition 3.6 of [M-R], we define

$$
E_{A}=A\left[v, v^{-1}\right]
$$

with degree $(v)=2(q-1)$ and give it the structure of a $V_{A}$ algebra via the map

$$
\phi^{\prime}: V_{A} \rightarrow E_{A}, \quad v_{i} \mapsto \begin{cases}v & \text { if } i=1, \\ 0 & \text { if } i>1 .\end{cases}
$$

We also define

$$
E_{A} T=E_{A} \otimes_{V_{A}} V_{A} T \otimes_{V_{A}} \dot{E}_{A}
$$

which forms with $E_{A}$, a Hopf algebroid. There is by extension a map of Hopf algebroids

$$
\left(\phi^{\prime}, \Phi^{\prime}\right):\left(V_{A}, V_{A} T\right) \rightarrow\left(E_{A}, E_{A} T\right) .
$$

It is $E_{A} T$ which we wish to describe. For this we will require the auxiliary Hopf algebroid $\left(K\left[v, v^{-1}\right], K\left[u, u^{-1}, v, v^{-1}\right]\right)$. The structure maps here are given by

$$
\eta_{R}(v)=v, \quad \eta_{L}(v)=u, \quad \psi(u)=u \otimes 1, \quad \psi(v)=1 \otimes v, \quad c(u)=v .
$$


To describe $E_{A} T$ we will examine its image in $K\left[u, u^{-1}, v, v^{-1}\right]$ under the canonical map $E_{A} T \rightarrow E_{A} T \otimes K$ and show that this map is injective. We will concentrate first on identifying the image of this map, and postpone the proof of injectivity to the end of $\S 2$. Since the image of this map is the same as that of the composition

$$
V_{A} T \rightarrow E_{A} T \rightarrow E_{A} T \otimes K=K\left[u, u^{-1}, v, v^{-1}\right]
$$

it is this that we study, i.e. the Hopf algebroid map

$$
(\phi, \Phi):\left(V_{a}, V_{A} T\right) \rightarrow\left(K\left[v, v^{-1}\right], K\left[u, u^{-1}, v, v^{-1}\right]\right) .
$$

Recall $[\mathbf{R} 1,2.8]$ that

$$
V_{A} T=V_{A}\left[t_{1}, t_{2}, \ldots\right] \quad \text { where } \operatorname{deg}\left(t_{n}\right)=2\left(q^{n}-1\right) .
$$

To describe the image of $\Phi$ we will obtain a recursive formula for $\Phi\left(t_{n}\right)$ in terms of $\Phi\left(t_{j}\right)$ for $j<n$. To do this we must first determine the value of the unique extension of $\phi$

$$
\bar{\phi}: V_{A} \otimes K \rightarrow K\left[v, v^{-1}\right]
$$

on the coefficients of the logarithm of the formal $A$-module.

LEMMA 1. If the log of the A-typical formal A-module is $\sum \lambda_{i} x^{q^{i}}$ then

$$
\bar{\phi}\left(\lambda_{n}\right)=v^{\left(q^{n}-1\right) /(q-1)} / \pi^{n} .
$$

ProOF. We have, from $[\mathbf{R 1}, 2.9]$, the formula

$$
\pi \lambda_{n}=\sum_{0 \leq i<n} \lambda_{i} v_{n-i}^{q^{i}} \text {. }
$$

Applying $\bar{\phi}$ we obtain

$$
\pi \bar{\phi}\left(\lambda_{n}\right)=\bar{\phi}\left(\lambda_{n-1}\right) \cdot v^{q^{n-1}}
$$

and the result follows.

PROPOSITION 2. The degree 0 component of the image of $\Phi$ is generated over $A\left[w, w^{-1}\right]$ by the set of polynomials $\left\{g_{n} \mid n=0,1,2, \ldots\right\}$ which are determined by the recursive formula

$$
g_{n}=\sum_{i=0}^{n-1} \frac{1}{\pi^{i+1}}\left(g_{n-i-1}^{q^{i}} \cdot w^{q^{n-1}}-g_{n-i-1}^{q^{i+1}}\right) .
$$

Here we have used the notation $w=v / u$.

PROOF. Starting with the same formula as in the proof above and applying $\eta_{R}$ yields

$$
\pi \eta_{R}\left(\lambda_{n}\right)=\sum_{0 \leq i<n} \eta_{R}\left(\lambda_{i}\right) \eta_{R}\left(v_{n-i}\right)^{q^{i}}
$$

or

$$
\pi \cdot \sum_{i=0}^{n} \eta_{L}\left(\lambda_{i}\right) \cdot t_{n-i}^{q^{i}}=\sum_{i=0}^{n-1} \sum_{j=0}^{i} \eta_{L}\left(\lambda_{j}\right) t_{i-j}^{q^{i}} \eta_{R}\left(v_{n-i}\right)^{q^{i}} .
$$

Applying $\Phi$ to both sides of this we obtain

$$
\pi \cdot \sum_{i=0}^{n} \phi\left(\eta_{L}\left(\lambda_{i}\right)\right) \Phi\left(t_{n-i}\right)^{q^{i}}=\sum_{i=0}^{n-1} \phi\left(\eta_{L}\left(\lambda_{i}\right)\right) \Phi\left(t_{n-i-1}\right)^{q^{i}} v^{q^{n-1}}
$$


since $\Phi\left(\eta_{R}\left(v_{n-i}\right)\right)=0$ unless $n-i=1$. This can be rewritten as

$$
\pi \cdot \sum_{i=0}^{n} \frac{1}{\pi^{i}} u^{\left(q^{i}-1\right) /(q-1)} \Phi\left(t_{n-1}\right)^{q^{i}}=\sum_{i=0}^{n-1} \frac{1}{\pi^{i}} u^{\left(q^{i}-1\right) /(q-1)} \Phi\left(t_{n-i-1}\right)^{q^{i}} \cdot v^{q^{n-1}} .
$$

To analyze this further we introduce the notation

$$
g_{i}=\Phi\left(t_{i}\right) \cdot u^{-\left(q^{i}-1\right) /(q-1)}
$$

Making this substitution we obtain, after some computation

$$
\sum_{i=0}^{n} \frac{1}{\pi^{i}} g_{n-i}^{q^{i}}=\sum_{i=0}^{n-1} \frac{1}{\pi^{i+1}} g_{n-i-1}^{q^{i}} \cdot w^{q^{n}-1} .
$$

Solving this for $g_{n}$ yields the result.

2. Integral valued polynomials and the image of $\Phi$. To analyze further the image of $\Phi$, we introduce certain subalgebras of the algebra Laurent polynomials:

$$
\begin{aligned}
& C=\left\{f \in K\left[w, w^{-1}\right] \mid f(1+\pi A) \subseteq A\right\}, \\
& B=C \cap K[w], \\
& \bar{B}=\{f \in K[x] \mid f(A) \subseteq A\} .
\end{aligned}
$$

Note that the algebras $B$ and $\bar{B}$ are isomorphic via the unique map of algebras sending $w$ to $1+\pi x$. In view of our observations in the preceding section concerning the polynomials $\left\{g_{n}\right\}$, showing that $(\operatorname{Im} \Phi)_{0} \subseteq C$ is equivalent to showing that $g_{n} \in$ $B$ or, writing $h_{n}(x)=g_{n}((x-1) / \pi)$, that $h_{n} \in \bar{B}$. In terms of these polynomials the recurrence formula of Proposition 2 becomes

$$
h_{n}=\sum_{i=0}^{n-1} \frac{1}{\pi^{i+1}}\left(h_{n-i-1}^{q^{i}}(\pi x+1)^{q^{n-1}}-h_{n-i-1}^{q^{i+1}}\right) .
$$

What we will show is

LEMMA 3. If $h \in \bar{B}, i<n$, then

$$
\frac{1}{\pi^{i+1}}\left(h^{q^{i}} \cdot(\pi x+1)^{q^{n-1}}-h^{q^{i+1}}\right) \in \bar{B} .
$$

Proof. We begin by noting two facts about $A$. First, if $u$ is a unit in $A$, then $u^{q-1} \equiv 1 \bmod \pi$. This is because the group of units modulo $\pi$ has order $q-1$. Second, the binomial theorem implies that if $y \equiv 1 \bmod \pi^{\nu}$ then $y^{q} \equiv 1 \bmod \pi^{\nu+1}$. It follows from this, by induction, that for any $x \in A$,

$$
(1+x \pi)^{q^{n-1}} \equiv 1 \quad \bmod \pi^{i+1}
$$

for any $i<n$.

From these two facts we see that for any $x \in A$,

$$
\begin{aligned}
h^{q^{i}} \cdot(\pi x+1)^{q^{n-1}}-h^{q^{i+1}} & \equiv h^{q^{i}}(x)-h^{q^{i+1}}(x) \bmod \pi^{i+1} \\
& \equiv h^{q^{i}}(x)\left(1-h^{(q-1) q^{i}}(x)\right) \bmod \pi^{i+1} \\
& \equiv 0 \quad \bmod \pi^{i+1} \cdot \quad
\end{aligned}
$$

We next examine the algebras $B$ and $\bar{B}$ more closely using techniques developed by G. Pólya and A. Ostrowski, published in $1919[\mathbf{O}, \mathbf{P}]$. The results in these papers 
are stated for the case of the ring of integers in an algebraic number field, but, as remarked in $[\mathbf{C}]$, they all extend directly to the case of any Dedekind ring with finite residue fields, including the discrete valuation ring, $A$, that we are concerned with. Our aim is to develop a way of recognizing a generating set for these algebras (which we will apply to $\left\{g_{n}\right\}$ or $\left\{h_{n}\right\}$ ). in $A$.

DEFINITION 4. (i) Let $\left\{p_{i} \mid i=0, \ldots, q-1\right\}$ be a complete set of residues for $\pi$

(ii) Let $\left\{\alpha_{i} \mid i=0,1,2, \ldots\right\}$ and $\left\{\bar{\alpha}_{i} \mid i=0,1,2, \ldots\right\}$ be subsets of $A$ defined by

$$
\bar{\alpha}_{n}=\sum_{i=0}^{k} p_{c_{i}} \pi^{i} \quad \text { and } \quad \alpha_{n}=1+\pi \bar{\alpha}_{n}
$$

if the expression of the integer $n$ in base $q$ is $n=\sum_{i=0}^{k} c_{i} q^{i}$.

(iii) Let $\psi: Z^{+} \rightarrow Z^{+}$be defined by

$$
\psi(n)=\sum_{i=1}^{k}\left[\frac{n}{q^{i}}\right]
$$

We can make the following observations concerning these definitions. First, the elements $\left\{\bar{\alpha}_{i}\right\}_{i=0}^{q^{k}-1}$ form a complete set of residues for $\pi^{k}$ in $A$ for any positive integer $k$. Next the elements $\left\{\alpha_{i}\right\}_{i=0}^{q^{k}-1}$ form a complete set of residues $\bmod \pi^{k+1}$ of those elements of $A$ congruent to $1 \bmod \pi$. Finally, the function $\psi$ could equally well be defined by the formula

$$
\psi(n)=\left(n-\sum_{i=0}^{k} c_{i}\right) /(q-1) .
$$

Given these observations, we may define some polynomials which form bases for $B$ and $\bar{B}$.

DEFINITION 5. Let $\hat{g}_{0}(w)=1=\bar{g}_{0}(x)$ and, for $n>0$,

$$
\hat{g}_{n}(w)=\prod_{i=0}^{n-1} \frac{w-\alpha_{i}}{\pi^{n+\psi(n)}}
$$

and

$$
\bar{g}_{n}(x)=\prod_{i=0}^{n-1} \frac{x-\bar{\alpha}_{i}}{\pi^{\psi(n)}}
$$

Proposition $6[\mathbf{P}, \mathbf{O}] . \quad$ (i) $\left\{\bar{g}_{n} \mid n=0,1,2, \ldots\right\}$ is a basis for $\bar{B}$ as an $A$ module.

(ii) $\left\{\hat{g}_{n} \mid n=0,1,2, \ldots\right\}$ is a basis for $B$ as an $A$-module.

PrOOF. In view of the isomorphism $B \simeq \bar{B}$ mentioned at the beginning of this section it suffices to prove (i).

That $\bar{g}_{n} \in \bar{B}$ for all $n$ follows from the fact that if $a \in A$, then

$$
\prod_{i=0}^{n-1} a-\bar{\alpha}_{i} \equiv 0 \quad \bmod \pi^{\psi(n)} .
$$

This can be found in [P, p. 106]. 
To show that the polynomials $\left\{\bar{g}_{n} \mid n=0,1, \ldots\right\}$ span $\bar{B}$, we require the additional fact from [P, Satz IV], that if $f \in \bar{B}$ is of degree $n$ then $\pi^{\psi(n)} \cdot f \in A[x]$. Thus, since the leading term of $\bar{g}_{n}$ is $1 / \pi^{\psi(n)}, f$ can be expressed as a linear combination of $\bar{g}_{0}, \ldots, \bar{g}_{n}$. Linear independence follows from the fact that degree $\left(\bar{g}_{n}\right)=n$.

To deduce information about the multiplicative structure of these algebras from this result it is convenient to introduce some auxiliary polynomials which yield different bases for $B$ and $\bar{B}$. Let $\left\{f_{i}(x) \mid i=0,1, \ldots\right\}$ be the polynomials given recursively by

$$
\begin{aligned}
f_{0}(x) & =x, \\
f_{1}(x) & =\left(x^{q}-x\right) / \pi, \\
f_{n+1}(x) & =f_{1} \circ f_{n}(x) .
\end{aligned}
$$

Also, if $n=\sum_{i=0}^{k} c_{i} q^{i}$ let

$$
\bar{f}_{n}(x)=\prod_{i}\left(f_{i}(x)\right)^{c_{i}} \quad \text { and } \quad \hat{f}_{n}(x)=\bar{f}\left(\frac{w-1}{\pi}\right) .
$$

PROPOSITION 7. The polynomials $\left\{\bar{f}_{n} \mid n=0,1,2, \ldots\right\}$ and $\left\{\hat{f}_{n} \mid n=0,1,2, \ldots\right\}$ are bases for the algebras $\bar{B}$ and $B$ respectively. The polynomials $\left\{f_{n} \mid n=0,1,2, \ldots\right\}$ are a generating set for $\bar{B}$.

PROOF. It suffices to prove the first of these three assertions. Since $\sum_{i=0}^{k} c_{i} \psi\left(q^{i}\right)$ $=\psi(n)$ the leading coefficient of $\bar{f}_{n}$ is $1 / \pi^{\psi(n)}$. Thus, for each $n$, the matrix expressing the polynomials $\left\{\bar{f}_{i} \mid i=0,1, \ldots, n\right\}$ as $A$ linear combinations of $\left\{\bar{g}_{i} \mid i=\right.$ $0,1, \ldots, n\}$ is triangular with units of $A$ along its diagonal. It is, therefore, invertible over $A$ by Cramer's rule. The result follows.

We are now ready to return to studying $\operatorname{Im}(\Phi)_{0}$ and to show that it equals $C$. For this we show that the polynomials $\left\{h_{n}(x) \mid n=0,1, \ldots\right\}$ generate $\bar{B}$. This is sufficient since $B$ generated $C$ over $A\left[w^{ \pm 1}\right]$ and $B \cong \bar{B}$. Our approach is to compare this prospective generating set with the one for $\bar{B}$ constructed above.

DEFINITION 8 . Denote by $R_{n}$ the subalgebra of $\bar{B}$ generated by $\left\{f_{i} \mid i=\right.$ $0,1, \ldots, n\}$.

These subalgebras have the property

LEMMA 9. (i) If $f \in \bar{B}$, and $\operatorname{deg}(f)<q^{n+1}$, then $f \in R_{n}$.

(ii) If $k<q^{n+2}$ is given, then there exists $f \in R_{n}$ with the properties that $\operatorname{deg}(f)=k$, and that the leading coefficient of $f$ is $1 / \pi^{\psi(k)-1}$.

PROOF. The subgroup of $\bar{B}$ of polynomials of degree less than $q^{n+1}$ has as a basis $\left\{\bar{f}_{i} \mid i=1,2, \ldots, q^{n+1}-1\right\}$. Since each of these is constructed as a product of polynomials in $R_{n}$ the first assertion is clear. Some arithmetic with the function $\psi$ shows that if $k=\sum_{i=0}^{n+1} c_{i} q^{i}$ then

$$
f=\prod_{i=0}^{n-1}\left(f_{i}\right)^{c_{i}} \cdot f_{n}^{c_{n}+q \cdot c_{n+1}}
$$

has the property claimed in the second assertion.

We are now ready to prove the following proposition. 
PROPOSITION 10. $R_{n}$ is generated by $\left\{h_{i} \mid i=1,2, \ldots, n+1\right\}$.

PROOF. We begin with a result concerning the coefficients of $h_{n}$. Suppose that

$$
h_{n}=\sum_{i=0}^{\left(q^{n}-1\right) /(q-1)} a_{i, n} x^{n}
$$

and that $\gamma$ is the $\pi$-adic valuation on $K$ as in [R1, p. 340], i.e. $\gamma$ is the extension to $K$ of the usual valuation on the $p$-adic numbers. We claim that $\gamma\left(a_{i, n}\right) \geq-\psi\left(q^{n-1}\right) / e$ with strict inequality if $i>q^{n-1}$ and equality if $i=q^{n-1}$. We will prove this by induction on $n$. For $n=1$ the result follows from direct computation. Suppose the result holds for $h_{n}$ and consider $h_{n+1}$.

Note that $\psi$ has the property

$$
\psi\left(q^{n}\right)=q \cdot \psi\left(q^{n-1}\right)+1>q^{2} \psi\left(q^{n-2}\right)+2, \quad \text { etc. }
$$

Now $h_{n+1}$ is given by

$$
h_{n+1}=\sum_{i=0}^{n}\left(\frac{h_{n-i}^{q^{i}}(\pi x+1)^{q^{n}}}{\pi^{i+1}}-\frac{h_{n-i}^{q^{i+1}}}{\pi^{i+1}}\right)
$$

and the $\pi$-adic norm of the coefficients of $h_{n-i}^{q^{i}}(\pi x+1)^{q^{n}} / \pi^{i+1}$ are all greater than $-\left(q^{i} \psi\left(q^{n-i-1}\right)+(i+1)\right) / e$ while those of $h_{k-i}^{q^{i+1}} / \pi^{i+1}$ will all be greater than $-\left(q^{i+1} \cdot \psi\left(q^{n-i-1}\right)+(i+1)\right) / e$. The previous observation shows that all of these are strictly greater than $-\psi\left(q^{n}\right) / e$ except possibly the second when $i=0$. Thus our problem reduces to that of examining the $\pi$-adic valuations of the coefficients of $h_{n}^{q} / \pi=\sum b_{i} x^{i}$.

For the moment let us write $a_{i}$ in place of $a_{i, n}$. The coefficient $b_{i}$ is

$$
b_{i}=\frac{1}{\pi} \sum a_{i_{1}} \cdots a_{i_{q}}
$$

where the sum is over all $q$-tuples $I=\left(i_{1} \cdots i_{q}\right)$ with

$$
|I|=\sum_{i=1}^{q} i_{j}=i .
$$

For $i>q^{n+1}$ at least one of the $i_{j}$ is greater than $q^{n}$ and so by induction

$$
-\frac{1}{e}+\gamma\left(\Pi a_{i_{j}}\right)>-\left(\frac{1}{e}+\frac{q \cdot \psi\left(q^{n-1}\right)}{e}\right)=-\frac{\psi\left(q^{n}\right)}{e} .
$$

On the other hand, when $i=q^{n+1}$ there is exactly one $q$-tuple with the property $i \leq q^{n}$ for all $j$ namely $i_{j}=q^{n}, j=1, \ldots, q$.

In this case

$$
-\frac{1}{e}+\gamma\left(\Pi a_{i j}\right)=-\left(\frac{1}{e}+q \gamma\left(a_{q^{n}}\right)\right)=-\left(\frac{1}{e}+\frac{q \psi\left(q^{n-1}\right)}{e}\right)=-\frac{\psi\left(q^{n}\right)}{e} .
$$

Thus $\gamma\left(b_{q^{n+1}}\right)=-\psi\left(q^{n}\right) / e$.

To complete our proof of this proposition we again induct on $n$. It is clear by direct computation that $R_{1}$ is generated by $h_{1}$ and $h_{2}$. Suppose that $R_{n}$ is generated by $\left\{h_{i} \mid i=1,2, \ldots, n+1\right\}$ and consider $R_{n+1}$. The polynomial $h_{n+2}$ has 
degree $\left(q^{n+2}-1\right) /(q-1)<q^{n+2}$ and so, applying the previous lemma and the observation above repeatedly we can find $f \in R_{n}$ such that $h_{n+2}-f$ is of degree $q^{n+1}$. Furthermore, the $\pi$-adic norm of the leading coefficient of $h_{n+2}-f$ will be $-\psi\left(q^{n+1}\right) / e$, again by the observation above. Thus

$$
\operatorname{Span}\left\langle R_{n}, h_{n+2}\right\rangle=\operatorname{Span}\left\langle R_{n}, h_{n+2}-f\right\rangle=\operatorname{Span}\left\langle R_{n}, f_{n+1}\right\rangle=R_{n+1} .
$$

We thus have

COROLlary 11. $\operatorname{Im}(\Phi)_{0}=C$.

It only remains for us to establish the injectivity of $\Phi$. The kernel of

$$
\Phi: E_{A} T \rightarrow E_{A} T \otimes K=K\left[u, u^{-1}, v, v^{-1}\right]
$$

will consist of the torsion subgroup of $E_{A} T$. Since $E_{A} T$ is a module over the local ring $A$ the injectivity of $\Phi$ will follow from that of the induced map

$$
\Phi^{\prime}: E_{A} T /(\pi) \rightarrow\left(C \otimes E_{A}\right) /(\pi)
$$

PROPOSITION 12. $\Phi^{\prime}$ is an isomorphism.

PROOF. If we let $K_{A}(1)=F_{q}\left[v_{1}, v_{1}^{-1}\right]$ as in $[\mathbf{R} 1]$, then

$$
\left(E_{A} T\right) /(\pi) \simeq K_{A}(1) \otimes V_{A} T \otimes K_{A}(1)
$$

which is denoted $\Sigma_{A}(1)$ in $[\mathbf{R 1}]$. It has the presentation

$$
\Sigma_{A}(1)=K_{A}(1)\left[t_{i} \mid i=1,2, \ldots\right] /\left(v_{1} t_{i}^{q}-v_{1}^{q^{i}} t_{i}\right) .
$$

Its degree 0 component, which we willl denote $S_{A}(1)_{*}$ has the presentation

$$
S_{A}(1)_{*}=F_{q}\left[S_{i} \mid i=1,2, \ldots\right] /\left(s_{i}^{q}-s_{i}\right) .
$$

The map $\left(\Phi_{0}^{\prime}\right)$ sends $s_{i}$ to $g_{i}$ so it will suffice to show that $\left(\Phi_{0}^{\prime}\right)$ restricted to the subalgebra of $S_{A}(1)_{*}$ generated by $s_{1}, \ldots, s_{n}$ maps isomorphically to the subalgebra generated by $g_{1}, \ldots, g_{n}$. We first note that the inclusion $B \hookrightarrow C$ induces an isomorphism $B /(\pi) \rightarrow C /(\pi)$. This is because $(w-1) / \pi \in B$ and $C$, so that $w \equiv 1$ $\bmod (\pi)$.

Now the subalgebra of $S_{A}(1)_{*}$ generated by $s_{1}, \ldots, s_{n}$ is clearly of rank $q^{n}$ over $F_{q}$. Thus, since $\Phi^{\prime}$ is surjective, we need only compute the rank of the subalgebra of $B /(\pi)$ generated by $g_{1}, \ldots, g_{n}$ or, equivalently, that of the subalgebra of $\bar{B} /(\pi)$ generated by $h_{1}, \ldots, h_{n}$. This is $R_{n-1} /(\pi)$ which is also generated by $f_{0}, \ldots, f_{n-1}$. Since $\pi \cdot f_{i+1}=f_{i}^{q}-f_{i}$ we have $f_{i}^{q}=f_{i}$ in $R_{n-1} /(\pi)$ and so $R_{n-1} /(\pi)$ has rank $q^{n}$ also.

3. $\operatorname{Ext}_{E_{A} T}\left(E_{A}, E_{A}\right)$ and Ravenel's conjecture. The relation between our description of $E_{A} T$ in $\S 2$ and the group $\operatorname{Ext}_{V_{A} T}\left(V_{A}, V_{A}\right)$ occurring in the Ravenel conjecture involves three steps. First the computation of $\operatorname{Ext}_{E_{A} T}\left(E_{A}, E_{A}\right)$, second the relation of this to the chromatic spectral sequence of $[\mathbf{R} \mathbf{1}]$, and finally the analysis of the differentials in that spectral sequence. We consider these in order.

The computation of $\operatorname{Ext}_{E_{A} T}\left(E_{A}, E_{A}\right)$ proceeds as in [S, Propositions 19.1719.22], where the special case $K=Q$ is considered. The only property of $Q$ that is used is that it is a field. 
Proposition 13. $\operatorname{Ext}_{E_{A} T}^{1,2 k(q-1)}\left(E_{A}, E_{A}\right)=A / J_{k \cdot(q-1)}$.

PROOF. In general, for any Hopf algebra $H$ over a ring $R$ we have

$$
\operatorname{Ext}_{H}^{1, k}(R, R)=P_{k}(H) /\left(\eta_{L}-\eta_{R}\right)(R)
$$

where $P_{k}()$ denotes the degree $k$ primitives. In our case this becomes

$$
\operatorname{Ext}_{E_{A} T}^{1,2 k(q-1)}\left(E_{A}, E_{A}\right)=P_{k}\left(E_{A} T\right) /\left(\eta_{L}-\eta_{R}\right)\left(E_{A}\right)
$$

and 0 in dimensions not divisible by $2(q-1)$. The denominator is easily identified:

$$
\left(\eta_{L}-\eta_{R}\right)\left(E_{A}\right)=A \cdot\left(u^{k}-v^{k}\right) \text {. }
$$

For the numerator, first note that

$$
P_{k}\left(K\left[u, u^{-1}, v, v^{-1}\right]\right)=K \cdot\left(u^{k}-v^{k}\right) .
$$

Therefore

$$
P_{k}\left(E_{A} T\right)=A \cdot\left(\left(u^{k}-v^{k}\right) / \pi^{d(k)}\right)
$$

where $d(k)$ is the largest integer for which $\left(u^{k}-v^{k}\right) / \pi^{d(k)} \in E_{A} T$, i.e. $\left(w^{k}-1\right) / \pi^{d(k)} \in C$. From our description of $C$ in $\S 2$ we see that $\pi^{d(k)} \cdot A=$ $J_{k \cdot(q-1)}$.

We next recall the construction of the chromatic spectral sequence and relate the Ext group constructed above to groups which occur there. Define inductively a pair of sequences of $V_{A} T$ comodules $N_{A}^{i}$ and $M_{A}^{i}$ by:

$$
N_{A}^{0}=V_{A}, \quad M_{A}^{i}=\left(v_{i}^{A}\right)^{-1} V_{A} \otimes N_{A}^{i}, \quad N_{A}^{i+1}=M_{A}^{i} / N_{A}^{i} .
$$

These occur in the short exact sequences

$$
0 \rightarrow N_{A}^{i} \rightarrow M_{A}^{i} \rightarrow N_{A}^{i+1} \rightarrow 0
$$

which can be combined to give a resolution of $V_{A}$ over $V_{A} T$ :

$$
0 \rightarrow V_{A} \rightarrow M_{A}^{0} \rightarrow M_{A}^{1} \rightarrow \cdots
$$

If we write

$$
D_{1}^{s, t}=\operatorname{Ext}_{V_{A} T}^{t, *}\left(V_{A}, N_{A}^{s}\right) \quad \text { and } \quad E_{1}^{s, t}=\operatorname{Ext}_{V_{A} T}^{t, *}\left(V_{A}, M_{A}^{s}\right)
$$

then we obtain an exact couple:

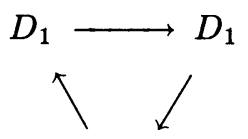

$E_{1}$

The spectral sequence associated to this couple is the chromatic spectral sequence, which converges to $\operatorname{Ext}_{V_{A} T}^{*, *}\left(V_{A}, V_{A}\right)$. Ravenel's conjecture is concerned with $\operatorname{Ext}_{V_{A} T}^{1, *}\left(V_{A}, V_{A}\right)$ which occurs as $E_{2}^{1,0}$ in this spectral sequence (since $E_{1}^{0,1}=0$ ). We therefore concentrate on

$$
E_{1}^{1,0}=\operatorname{Ext}_{V_{A} T}^{0, *}\left(V_{A}, M_{A}^{1}\right), \quad E_{1}^{2,0}=\operatorname{Ext}_{V_{A} T}^{0, *}\left(V_{A}, M_{A}^{2}\right)
$$

and the differential $d_{1}$ between these two groups, whose kernel is $E_{2}^{1,0}$.

The first of these groups is related to our previous computation by the following $v_{1}$ local change of rings theorem which holds for any $v_{1}$ local $V_{A} T$ comodule, but which we state only for the special case which we need. 
THEOREM 14. $\operatorname{Ext}_{V_{A} T}\left(V_{A}, M_{A}^{1}\right) \simeq \operatorname{Ext}_{E_{A} T}\left(E_{A}, E_{A} \otimes_{V_{A}} M_{A}^{1}\right)$.

The proof of this result is a straightforward generalization of Theorem 3.10 of [M-R].

Based on this theorem we can compute $E_{1}^{1,0}$.

COROLLARY 15.

$$
\operatorname{Ext}_{V_{A} T}^{0, *}\left(V_{A}, M_{A}^{1}\right) \simeq \begin{cases}K / A & \text { if } *=0 \\ \operatorname{Ext}_{E_{A} T}^{1, *}\left(E_{A}, E_{A}\right) & \text { otherwise. }\end{cases}
$$

ProOF. When the map $d_{0}: M_{A}^{0} \rightarrow M_{A}^{1}$ is tensored with $E_{A}$ it yields the exact sequence

$$
0 \rightarrow E_{A} \rightarrow E_{A} \otimes_{V_{A}} M_{A}^{0} \rightarrow E_{A} \otimes_{V_{A}} M_{A}^{1} \rightarrow 0
$$

which has associated to it a long exact sequence of Ext groups. Since the middle term in the short exact sequence is isomorphic to $E_{A} \otimes_{A} K$ whose Ext groups are given by

$$
\operatorname{Ext}_{E_{A} T}\left(E_{A}, E_{A} \otimes_{A} K\right)= \begin{cases}K & \text { if }(*, *)=(0,0), \\ 0 & \text { otherwise }\end{cases}
$$

the result follows.

The proof of this corollary provides us with a convenient representation for elements of $E_{1}^{1,0}$. If we take the cobar resolution over $E_{A} T$ of the groups in the short exact sequence, then we obtain the diagram:

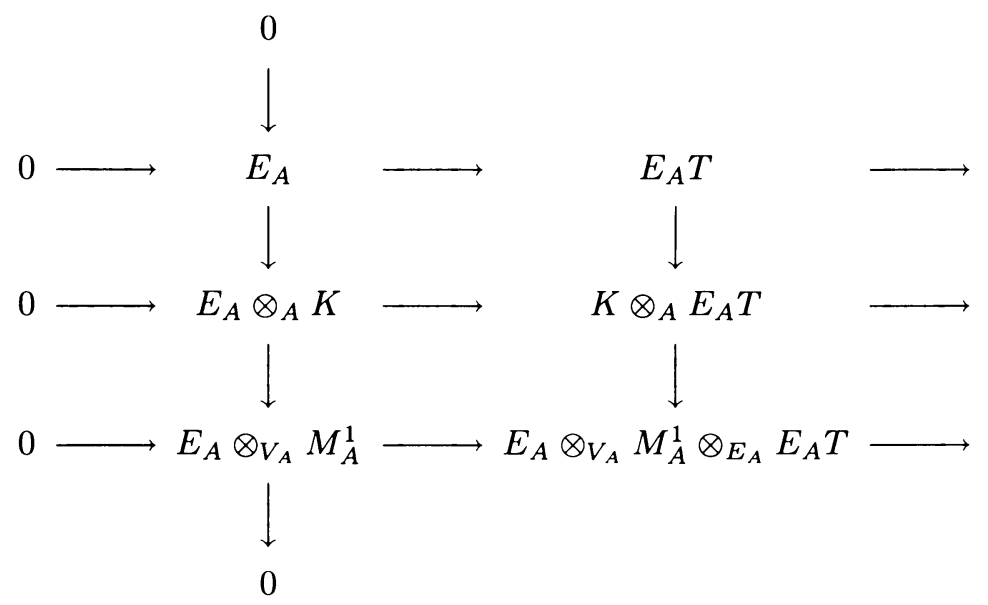

Using Theorem 14 and this diagram we see that we may represent an element of $\operatorname{Ext}_{V_{A} T}^{0, *}\left(V_{A}, M_{A}^{1}\right)$ by an element $x \in E_{A} \otimes_{A} K$ with the property that $\left(\eta_{L}-\eta_{R}\right)(x) \in$ $E_{A} T$. Such a representative is unique modulo $E_{A}$.

The final step in our consideration of Ravenel's conjecture is the study of the differential $d_{1}$ and the identification of its kernel. We first describe this kernel in terms of the representation given above for elements of $E_{1}^{1,0}$ and then do the necessary number theory to determine when this kernel is all of $E_{1}^{1,0}$.

Using the long exact Ext sequences associated to the short exact sequences

$$
0 \rightarrow N_{A}^{i} \rightarrow M_{A}^{i} \rightarrow N_{A}^{i+1} \rightarrow 0
$$


for $i=1,2$ and the fact that the differential $d_{1}$ is given by the composition

$$
\operatorname{Ext}_{V_{A} T}^{0, *}\left(V_{A}, M_{A}^{1}\right) \rightarrow \operatorname{Ext}_{V_{A} T}^{0, *}\left(V_{A}, N_{A}^{2}\right) \rightarrow \operatorname{Ext}_{V_{A} T}^{0, *}\left(V_{A}, M_{A}^{2}\right)
$$

we see that the kernel of $d_{1}$ is given by the image of $\operatorname{Ext}_{V_{A} T}^{0, *}\left(V_{A}, N_{A}^{1}\right)$ in $\operatorname{Ext}_{V_{A} T}^{0, *}\left(V_{A}, M_{A}^{1}\right)$. We can relate this to our earlier description of elements in $E_{1}^{1,0}$ using the following diagram whose rows are exact:

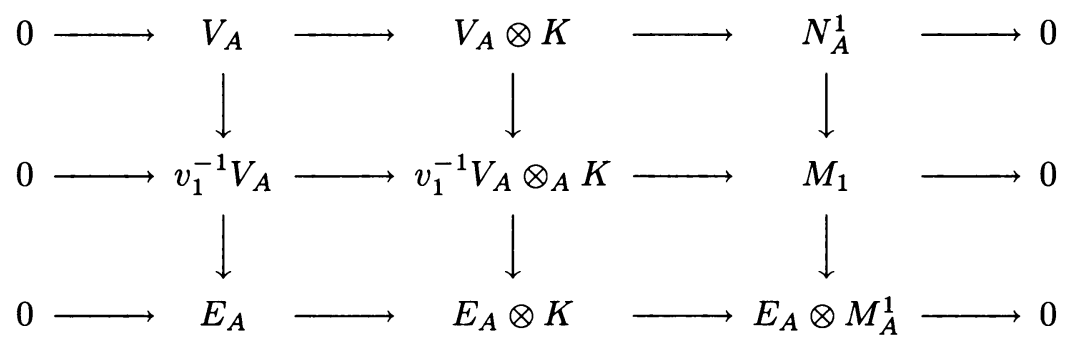

Tracing through this diagram we see that an element $x \in E_{A} \otimes K$ represents an element in the image of $\operatorname{Ext}_{V_{A} T}^{0, *}\left(V_{A}, N_{A}^{1}\right)$ if it is the image under the center vertical composition of an element $y \in V_{A} \otimes K$ with the property that $\left(\eta_{L}-\eta_{R}\right)(y) \in V_{A} T$. The content of Theorem 14 is that any $x \in E_{A} \otimes K$ representing an element of $E_{1}^{1,0}$ will be the image of an element $z \in v_{1}^{-1} V_{A} \otimes K$ with $\left(\eta_{L}-\eta_{R}\right)(z) \in v_{1}^{-1} V_{A} \otimes V_{A} T$. It will be the image of an element $y$ as above if the expression of $z$ as a Laurent polynomial in $v_{1}$ does not involve any negative powers of $v_{1}$ which are nontrivial modulo $V_{A}$.

Before we can describe this kernel more explicitly we require a more precise description of the arithmetic function $d(l)$ which determines the ideal $J_{*}$.

PROPOSITION 16. If $(m, p)=1$ then $d\left(m p^{k}\right)=d\left(p^{k}\right)$.

PROOF. $d$ is defined so that for any $a \in A$ with $a \equiv 1 \bmod \pi$ we have

$$
a^{p^{k}} \equiv 1 \bmod \pi^{d\left(p^{k}\right)} \text {. }
$$

Therefore

$$
a^{m p^{k}}=\left(a^{p^{k}}\right)^{m} \equiv 1 \bmod \pi^{d\left(p^{k}\right)}
$$

and so $d\left(m p^{k}\right) l \geq d\left(p^{k}\right)$.

On the other hand there exists $a \in A$ with $a \equiv 1 \bmod \pi$ for which

$$
a^{p^{k}} \not \equiv 1 \bmod \pi^{d\left(p^{k}\right)+1} \text {. }
$$

Using the binomial theorem we see that this implies that, since $(m, p)=1$,

$$
a^{m p^{k}}=\left(a^{p^{k}}\right)^{m} \not \equiv 1 \bmod \pi^{d\left(p^{k}\right)+1}
$$

and so $d\left(m p^{k}\right) \leq d\left(p^{k}\right)$ also.

Thus we can restrict our attention to the value of $d$ at powers of $p$. For this we will use the following result concerning congruences in $A$ from [Ha, p. 228].

LEMMA 17. If $x \in A$ is such that $x \equiv 1+a \pi^{\nu} \bmod \pi^{\nu+1}$ then

$$
x^{p}= \begin{cases}1+a^{p} \pi^{p \nu} \bmod \pi^{p \nu+1} & \text { if } \nu<e /(p-1), \\ 1+\left(a^{p}-\varepsilon a\right) \pi^{p \nu} \bmod \pi^{p \nu+1} & \text { if } \nu=e /(p-1), \\ 1-\varepsilon a \pi^{\nu+e} \bmod \pi^{\nu+e+1} & \text { if } \nu>e /(p-1),\end{cases}
$$

where $\varepsilon$ is the unit in $A$ for which $p=-\varepsilon \pi^{e}$. 
This lemma allows us to explicitly describe $d$ if $e /(p-1)$ is not a power of $p$.

Proposition 18. If $p^{l-1}<e /(p-1)<p^{l}$ then

$$
d\left(p^{k}\right)= \begin{cases}p^{k} & \text { if } k \leq l, \\ p^{l}+(k-1) e & \text { if } k>l .\end{cases}
$$

Proof. The lemma shows that, for $k \leq l d\left(p^{k}\right)=p \cdot d\left(p^{k-1}\right)$ and, for $k>l$ $d\left(p^{k}\right)=e+d\left(p^{k-1}\right)$.

If $e /(p-1)$ is a power of $p$ the situation is the same in low dimensions. Just as above we can show

Proposition 19. If $e /(p-1)=p^{l}$ and $k \leq l$ then $d\left(p^{k}\right)=p^{k}$.

The critical level is $k=(l+1)$ where the existence of $p$ th roots of unity becomes important.

Proposition 20. If $k>l$, then the following equation holds except in the case where $e=p^{l}(p-1), f=1$, and $A$ contains pth roots of unity:

$$
d\left(p^{k}\right)=p^{l}+(k-l) \cdot e .
$$

PROOF. The result will follow as above for all $k>l$ if we can establish it for the case $k=l+1$. If $x=1+a \pi \bmod \pi^{2}$ then, by the lemma,

$$
x^{p^{l+1}} \equiv 1+\left(a^{p l+1}-\varepsilon a^{p^{l}}\right) \pi^{p^{l+1}} \bmod \pi^{p^{l+1}+1} .
$$

Thus it suffices for us to show that there exists $a \in A$ which is not a solution of the congruence $\left(a^{p l+1}-\varepsilon a^{p^{l}}\right) \equiv 0 \bmod \pi$. To do this we count the number of solutions of this congruence and show that there are less than $p^{f}$ of them modulo $\pi$. Since the $p$ th power map is an automorphism of $A / \pi A$ any solution of this congruence is the $p^{l}$ th power of a (unique modulo $\pi$ ) solution of $a^{p}-\varepsilon a \equiv 0 \bmod \pi$. This congruence has either $p$ solutions or 1 solution modulo $\pi$ according to whether $A$ does or does not contain $p$ th roots of unity [Ha, p. 224].

Proposition 21. If $e /(p-1)=p^{l}, f=1$ and $A$ contains $p$ th roots of unity then $p^{l+1}<d\left(p^{l+1}\right) \leq p^{l+1}+p^{l}$.

PROOF. The left hand inequality follows from the previous proof. For the right hand one, note that

$$
\left(1+\pi^{2}\right)^{p^{l+1}} \equiv 1+\pi^{p^{l+1}+p^{l}} \quad \bmod \pi^{p^{l+1}+p^{l}+1} .
$$

REMARK 22. Using the description of the 1-units (units congruent to $1 \bmod \pi$ ) in $A$ given in [Ha, p. 242] the left-hand inequality above can be improved to $p^{l+1}+$ $p^{\mu-1} \leq d\left(p^{l+1}\right)$ if the group of roots of unity of $p$ th power order in $A$ has order $p^{\mu}$.

For $k>l+1$ the computation is as before.

PROPOSITION 23. If $d\left(p^{l+1}\right)=p^{l+1}+u$ then, for $k>l+1, d\left(p^{k}\right)=p^{l}+(k-$ l) $\cdot e+u$.

We can now complete our description of the kernel of $d_{1}$. Proposition 13 and the description of $d(l)$ given above give an upper bound for the order of this kernel. To identify cases in which this upper bound is attained we must construct specific elements in $E_{1}^{1.0}$. 
PROPOSITION 24. (i) If $(m, p)=1, p^{l} \leq e /(p-1)<p^{l+1}$ and $k \leq l$ then $\left(\eta_{L}-\eta_{R}\right)\left(v_{1}^{m p^{k}} / \pi^{p^{k}}\right) \in V_{A} T$.

(ii) If $(m, p)=1, p^{l} \leq e /(p-1)<p^{l+1}$ and $k>l$ then $\left(\eta_{L}-\eta_{R}\right)\left(v_{1}^{m p^{k}} / \pi^{p^{l}+(k-l) e}\right)$ $\in V_{A} T$.

PROOF. Since $\left(\eta_{R}\right)\left(v_{1}\right)=\left(v_{1}+\pi t_{1}\right)$ and so $\left(\eta_{L}-\eta_{R}\right)\left(v_{1}\right)=\left(-\pi t_{1}\right)$ both these results follow from computing the $\pi$-adic valuations of the coefficients of the binomial expansion of $\left(v_{1}+\pi t_{1}\right)^{m p^{k}}$.

Combining Proposition 20 with the description of elements of $\operatorname{Ker}\left(d_{1}\right)$ following Corollary 15 we see that we have

COROLlaRY 25. Except in the case where $e=p^{l}(p-1), f=1$, and A contains pth roots of unity the following equation holds:

$$
\operatorname{Ext}_{V_{A} T}^{1, n(q-1)}\left(V_{A}, V_{A}\right)=A / J_{n(q-1)} .
$$
$E_{1}^{1,0}$.

In the case excluded in Corollary 25 we can construct the following element of

Proposition 26. If $e /(p-1)=p^{l}, f=1, A$ contains $p$ th roots of unity and $u$ is as in Proposition 23 then

$$
\left(\eta_{L}-\eta_{R}\right)\left(\left(v_{1}^{p^{l+1}}\right)-\pi^{p^{l+1}}\left(v_{2} / v_{1}\right)^{p^{l}}\right) / \pi^{p^{l+1}+u} \in V_{A} T .
$$

Proof. Since $u \leq p^{l}$ it is sufficient for us to compute the numerator of this quotient modulo $\pi^{p^{l+1}}+p^{l}$. Expanding $\left(v_{1}+\pi t_{1}\right)^{p^{l+1}}$ we obtain

$$
\begin{aligned}
\left(\eta_{L}-\eta_{R}\right)\left(v_{1}\right)^{p^{l+1}} & \equiv\left(\begin{array}{c}
p^{l+1} \\
p^{l}
\end{array}\right) \pi^{p^{l}} t_{1}^{p^{l}} v_{1}^{p^{l}(p-1)}+\pi^{p^{l+1}} t_{1}^{p^{l}} \\
& \equiv p \pi^{p^{l}} t_{1}^{p^{l}} v_{1}^{p^{l}(p-1)}+\pi^{p^{l+1}} t_{1}^{p^{l}} \\
& \equiv \pi^{p^{+1}}\left(t_{1}^{p^{l+1}}-\varepsilon t_{1}^{p^{l}} v_{1}^{p^{l}(p-1)}\right) \quad \bmod \pi^{p^{l+1}+p^{l}} .
\end{aligned}
$$

On the other hand $\eta_{R}\left(v_{2} / v_{1}\right) \equiv\left(v_{2} / v_{1}\right)+t_{1}^{p}-t_{1} v_{1}^{p-1} \bmod \pi$ and so

$$
\left(\eta_{L}-\eta_{R}\right)\left(\pi^{p^{l+1}}\left(v_{2} / v_{1}\right)^{p^{l}}\right) \equiv \pi^{p^{l+1}}\left(t_{1}^{p^{l+1}}-t_{1}^{p^{l}} v_{1}^{p^{l}(p-1)}\right) \quad \bmod \pi^{p^{l+1}+p^{l}} .
$$

The result now follows from the observation that $\varepsilon \equiv 1 \bmod \pi^{u}$ which can be established by expanding $(1+\pi)^{p^{l+1}}$ and noting that it must be congruent to $1 \bmod \pi^{p^{l+1}+u}$.

Since the element $\left(\left(v_{1}^{p^{l+1}} / \pi^{p^{l+1}+1}\right)-\left(v_{2} / v_{1}\right)^{p^{l}} / \pi\right)$ contains negative powers of $v_{1}$ which are nonzero modulo $V_{A} T$ we have constructed an element of $E_{1}^{1,0}$ which is not in the kernel of $d_{1}$. We have, therefore

COROLlary 27. If $f=1, e /(p-1)=p^{l}$, and $A$ contains pth roots of unity then

$$
\operatorname{Ext}_{V_{A} T}^{1, p^{l+1}(p-1)}\left(V_{A}, V_{A}\right)=A /\left(\pi^{p^{l+1}}\right) \neq A / J_{p^{l+1}(p-1)} .
$$

We can also take powers of the elements constructed in Proposition 26 to obtain elements of $\operatorname{Ext}^{1, n(p-1)}$ if $p^{l+1}$ divides $n$. These powers do not contain any negative powers of $v_{1}$ which are nontrivial modulo $V_{A} T$. Thus we can complete our description of $\operatorname{Ext}_{V_{A} T}^{1, *}\left(V_{A}, V_{A}\right)$ : 
COROLlary 28. If $f=1, e /(p-1)=p^{l}$, and $A$ contains $p$ th roots of unity, then except in the case $n=p^{l+1}$,

$$
\operatorname{Ext}_{V_{A} T}^{1, n(p-1)}\left(V_{A}, V_{A}\right)=A / J_{n(p-1)} .
$$

\section{REFERENCES}

[AHS] J. F. Adams, A. S. Harris and R. M. Switzer, Hopf algebras of co-operations for real and complex $K$-theory, Proc. London Math. Soc. 23 (1971), 385-408.

[C] P. J. Cahen, Polynomes a valeurs entieres, Canad. J. Math. 24 (1972), 747-754.

[CF] J. Cassels and A. Fröhlich, Algebraic number theory, Academic Press, New York, 1967.

[Ha] H. Hasse, Number theory, Springer-Verlag, New York, 1980.

$[\mathbf{H z}]$ M. Hazewinkel, Formal groups and applications, Academic Press, New York, 1973.

[MR] H. R. Miller and D. C. Ravenel, Morava stabilizer algebras and the localization of Novikov's $E_{2}$ term, Duke Math. J. 44 (1977), 433-447.

[O] A. Ostrowski, Über Ganzwertige Polynome in Algebraischen Zahlkorper, J. Reine Angew. Math. (Crelle) 149 (1919), 117-124.

[P] G. Polya, Über Ganzwertige Polynome in Algebraischen Zahlkörper, J. Reine Angew. Math. (Crelle) 149 (1919), 97-116.

[R1] D. C. Ravenel, Formal A-modules and the Adams-Novikov spectral sequence, J. Pure Appl. Math. 32 (1984), 327-345.

[R2] _ The structure of Morava stabilizer algebras, Invent. Math 37 (1976), 109-120.

[S] R. M. Switzer, Algebraic topology-homotopy and homology, Grundlehren Math. Wiss., Band 212, Springer-Verlag, Berlin and New York, 1975.

Department of Mathematics, Statistics and Computing Science, Dalhousie University, Halifax, Nova SCOTia, CANADA 